\title{
Immature granulocyte percentage for prediction of sepsis in severe burn patients: a machine leaning-based approach
}

\author{
Kibum Jeon ${ }^{1}$, Nuri Lee ${ }^{2 *}$, Seri Jeong ${ }^{2}$, Min-Jeong Park ${ }^{2}$ and Wonkeun Song ${ }^{2}$
}

\begin{abstract}
Background: Of the existing sepsis markers, immature granulocytes (IG) most frequently reflect the presence of an infection. The importance of IG as an early predictor of sepsis and bacteremia is evaluated differently for each study. This study aimed to evaluate the effectiveness of the Sysmex XN series'IG\% as an independent prognostic indicator of sepsis using machine learning.

Methods: A total of $2465 \mathrm{IG} \%$ results from 117 severe burn patients in the intensive care unit of one institution were retrospectively analyzed. We evaluated the IG\% for sepsis using the receiver operating characteristic, logistic regression, and partial dependence plot analyses. Clinical characteristics and other laboratory markers associated with sepsis, including WBC, procalcitonin, and C-reactive protein, were compared with the IG\% values.
\end{abstract}

Results: Twenty-six of the 117 patients were diagnosed with sepsis. The median IG\% value was 2.6\% (95\% Cl: 1.4-3.1). The area under the receiver operating characteristic curve was 0.77 (95\% Cl: $0.78-0.84)$ and the optimal cut-off value was $3 \%$, with a sensitivity of $76.9 \%$ and specificity of $68.1 \%$. The partial dependence plot of IG\% on predicting sepsis showed that an IG\% < 4\% had low predictability, but increased thereafter. The interaction plot of IG\% and C-reactive protein showed an increase in sepsis probability at an IG\% of $6 \%$ and C-reactive protein of $160 \mathrm{mg} / \mathrm{L}$.

Conclusions: IG\% is moderately useful for predicting sepsis. However, since it can be determined from routine laboratory test results and requires no additional intervention or cost, it could be particularly useful as an auxiliary marker.

Keywords: Sepsis, Immature granulocytes, Machine learning, Severe burn

\section{Background}

Burns are complex traumatic injuries that require appropriate treatment, including surgical intervention in the acute phase. Since the skin is the first barrier to infection, burn patients are constantly exposed to inflammatory mediators while the wound remains exposed. As a result, severe burn patients often develop persistent systemic

\footnotetext{
${ }^{*}$ Correspondence: nurilee822@hallym.or.kr

${ }^{2}$ Department of Laboratory Medicine, Hallym University Kangnam Sacred Heart Hospital, Hallym University College of Medicine, 22, Singil-ro 1, Yeongdeungpo-gu, Seoul 07440, South Korea

Full list of author information is available at the end of the article
}

inflammatory response syndrome (SIRS), which can lead to sepsis [1].

Sepsis, which occurs when SIRS is accompanied by an infection, is common in burn patients. This is due to the dysregulation of the host's response to infection and can be a major risk factor for mortality. The early diagnosis of sepsis before progression to organ dysfunction has a decisive effect on the clinical course and outcomes of patients with severe sepsis [2]. However, since sepsis is a clinical syndrome involving many heterogeneous conditions and not a final diagnosis, there is no definitive model for the diagnosis of sepsis [3]. Furthermore, patients with severe burns have persistent tachycardia, tachypnea, and fever 
due to SIRS, making it difficult to predict sepsis based on clinical signs. Thus, biomarkers are of high clinical importance for predicting sepsis in burn patients.

Immature granulocytes (IG) are recently-produced granulocytes that have been released into the circulation. While IG are normally absent from peripheral blood, they increase in conditions such as bacterial sepsis, inflammation, trauma, cancer, steroid therapy, and myeloproliferative diseases $[4,5]$. Previous studies have shown that the IG percentage (IG\%) is clinically significant for the early detection of bacterial infections or in predicting the risk of sepsis in the intensive care unit. However, most studies have been conducted on pediatric patients, and few have studied adult burn patients [6-8]. In addition, the importance of IG as an early predictor of sepsis has been evaluated differently in several studies. Differences in patient groups or confounding factors could contribute to these differences $[9,10]$. Therefore, it is necessary to clarify whether the IG\% could be an independent indicator of prognosis compared to existing sepsis indicators, especially for severe burn patients.

In this study, we evaluated the effectiveness of the IG\% as an independent predictor of sepsis in patients with severe burns using the IG index of the Sysmex XN (Sysmex, Kobe, Japan), which can objectively calculate the IG\%. Moreover, as machine learning has recently received attention as an effective prediction tool that may improve the accuracy of predictions, we used a machine learning-based prediction model.

\section{Methods}

\section{Patients}

This study included 117 patients treated in the burn intensive care unit (BICU) at the Burn Center, Hallym University Hangang Sacred Hospital, Seoul, Republic of Korea between July and December 2017. Data collected for each patient were as follows: age, sex, percentage of total body surface area burned (\%TBSA), cause of burn injury, and presence of inhalation injury. All patients with burns were newly diagnosed, with no history of underlying disease. The study was approved by the institutional review board of the Hallym University Hangang Sacred Hospital (IRB no. HG2018-069), which waived the requirement for written informed consent owing to the observational and anonymized nature of the study. The study was performed in accordance with the guidelines of the Declaration of Helsinki.

\section{Diagnosis of sepsis in burn patients}

We classified a sepsis group according to the diagnostic criteria for sepsis in burn patients of the American Burn Society (Table 1) [11]. Patients who did not belong to this group were classified into the non-sepsis group. The date of diagnosis of sepsis was designated as the first day that satisfies the diagnostic criteria in Table 1 during inpatient treatment in BICU.

\section{Measurement of IG and biomarkers associated with sepsis}

For the complete blood count, which included the IG\% and IG count, were analyzed by K3-EDTA (BD, Franklin Lakes, NJ) anticoagulated venous whole blood samples using the $\mathrm{XN}-1000$ automated hematology analyzer (Sysmex, Kobe, Japan). For measure IG, the white cell differential channel (WDF) was used after applying a specific lysing agent (Lysercell WDF), and differentiation was determined according to granularity (side scatter) and nucleic acid content (side fluorescence by the Fluorocell WDF reagent). The IG cluster was found right above the neutrophil cluster in the scatter/fluorescence of the biparametric histogram side (Fig. 1). The IG\% was calculated using the following formula: (particle count in IG zone/particle count in WBC zone) $\times 100$. The IG\% included promyelocytes, myelocytes, and metamyelocytes, while blasts and band cells were not included [12].

Biomarkers associated with sepsis, including procalcitonin, C-reactive protein (CRP), and lactate, were compared to the IG\%. All samples were analyzed using SST-II (BD, Franklin Lakes, NJ, USA) serum samples.

Table 1 Diagnostic criteria for sepsis in burn patients

Culture positive infection and at least three of the following:

1. Fever $>39^{\circ} \mathrm{C}$

2. Hypothermia $\left(<36.5^{\circ} \mathrm{C}\right)$

3. Progressive tachycardia ( $>110$ beats per min)

4. Progressive tachypnea ( $>25$ breaths per minute not ventilated or minute ventilation $>12 \mathrm{~L} /$ min ventilated)

5. Thrombocytopenia $(<100,000 / \mu \mathrm{l})$

6. Hyperglycemia, in the absence of preexisting diabetes mellitus (untreated plasma glucose $>200 \mathrm{mg} / \mathrm{dl}$ or $>7$ units of insulin/h intravenous drip or significant resistance to insulin, $>25 \%$ increase in insulin requirement over $24 \mathrm{~h}$ );

7. Inability to continue enteral feedings $>24 \mathrm{~h}$ (abdominal distension or high gastric residuals, residuals two times feeding rate or uncontrollable diarrhea, $>2500 \mathrm{ml} /$ day) 


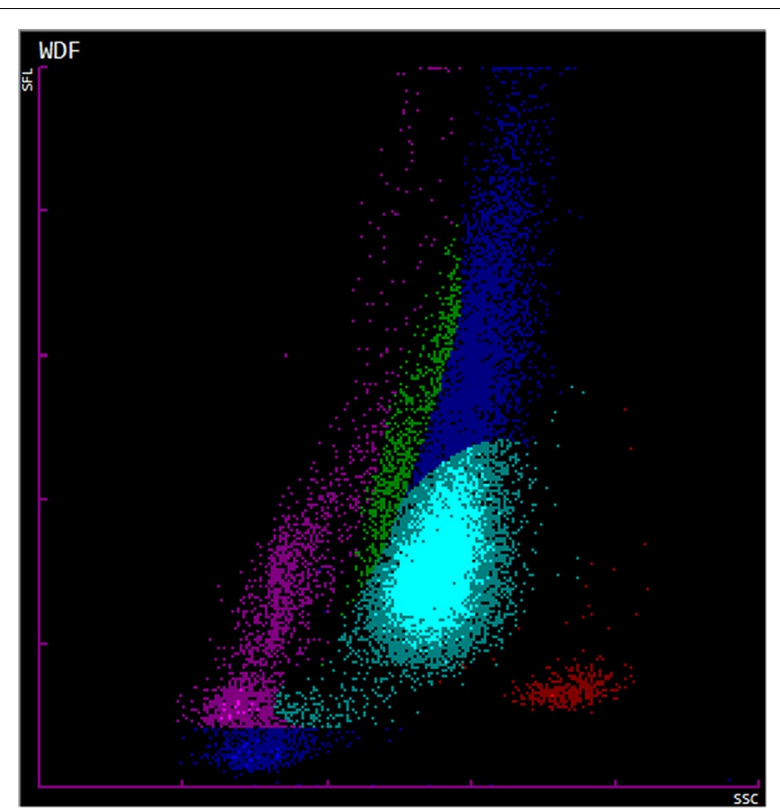

Fig. 1 White cell differential channel (WDF) scattergram of a burn patient with sepsis, IG\% of $17.1 \%$. The WDF channel differentiates and counts lymphocytes (purple), monocytes (green), eosinophils (red), mature neutrophils (cyan), Immature Granulocytes (blue). Signal fluorescence intensity (SFL) was used to separate cells according to the DNA/RNA content and determine the immaturity and activation of the examined cells (y axis). Forward scattering (FSC, $z$ axis) and side scattering (SSC, $x$ axis) indicate cell size and the complexity of the intracellular structure, respectively

Procalcitonin and CRP levels were measured using a Cobas c702 (Roche Diagnostics, Mannheim, Germany), and lactate was analyzed using an Ultra-M Analyzer (Nova Biomedical, Waltham, MA, USA). The highest IG\% and biomarker values before or at the same time point of sepsis for each patient were used.

\section{Statistical analyses}

Statistical analyses were performed using the SPSS statistics program version 24 (IBM Corporation, New York, NY) and MedCalc version 18 (MedCalc Software, Mariakerke, Belgium). Quantitative variables are presented as the median and interquartile range (IQR: 25th-75th percentiles). The Mann-Whitney test was applied to compare nonparametric quantitative variables between the two groups, whereas the Kruskal-Wallis test was used to compare more than two groups. The chi-square test was used to compare the distribution of the two groups.

Receiver operating characteristic (ROC) curves were used to compare the IG\% and other parameters between the sepsis and non-sepsis patient groups. The IG\% diagnostic cut-off value with the best combined sensitivity and specificity was also determined. The area under the curve (AUC) and 95\% confidence interval (CI) were calculated for each plot.

A logistic regression analysis was performed to evaluate the predictive value of the IG\% for sepsis. Univariate and multivariate regression analyses were performed for the biomarkers and risk factors. Statistical significance was set at $P<0.05$.

\section{Machine learning approach}

The machine learning interpretability techniques were analyzed using the R-based pdp package (version 3.6.3). Partial dependence plots (PDP) and individual conditional expectations (ICE) are used to interpret and visualize the partial dependence of the probability of developing an outcome (sepsis) on a predictor (IG\%, biomarkers, and risk factors). Both single-predictor and multi-predictor PDP were performed for this study. For the multi-predictor PDP, the grid.arrange function was used to display a color map for level plots. ICE curves were displayed on the same plot with the average PDP using the ggplot2 package and centered on the zero base. The schematic diagram of machine learning process is provided in the Additional file 1: Fig. S1.

\section{Results}

Patient characteristics in the sepsis and non-sepsis groups The demographic characteristics of the 117 patients, 26 of which were in the sepsis group $(22.2 \%)$ and 91 in the non-sepsis group (77.8\%), are summarized in Table 2 . The \%TBSA, presence of inhalation burns, length of stay in the BICU, death rates and positive rate of blood culture were significantly different between the two groups $(\mathrm{P}<0.001)$.

\section{Utility of biomarkers for predicting sepsis WBC and absolute neutrophil count}

The median [IQR] WBC count in the sepsis group and non-sepsis group was $10.4 \times 10^{9} / \mathrm{L}\left[4.5-15.2 \times 10^{9} / \mathrm{L}\right]$ and $12.0 \times 10^{9} / \mathrm{L}\left[8.4-16.0 \times 10^{9} / \mathrm{L}\right]$, respectively. The absolute neutrophil count was $8.1 \times 10^{9} / \mathrm{L}\left[3.8-11.7 \times 10^{9} / \mathrm{L}\right]$ and $9.4 \times 10^{9} / \mathrm{L}\left[5.7-12.2 \times 10^{9} / \mathrm{L}\right]$, respectively. While both were higher in the non-sepsis group, the differences were not statistically significant.

\section{IG\% and IG count}

In the sepsis group, the median [IQR] IG\% value was $6.4 \%$ [3.1-11.6\%], which was significantly higher than that in the non-sepsis group (1.4\% [0.6-4.4\%], $P<0.001)$. The IG count was also significantly higher in the sepsis group $\left(0.64 \times 10^{9} / \mathrm{L}\left[0.30-2.02 \times 10^{9} / \mathrm{L}\right], P<0.001\right)$ than in the non-sepsis group $\left(0.21 \times 10^{9} / \mathrm{L}\left[0.06-0.63 \times 10^{9} / \mathrm{L}\right]\right)$. 
Table 2 Demographic and laboratory characteristics of the patients

\begin{tabular}{|c|c|c|c|}
\hline & All $(\mathrm{N}=117)$ & Sepsis $(N=26)$ & Non-sepsis $(\mathrm{N}=91)$ \\
\hline Age, year & $52(41.5-60.0)$ & $49.5(28.0-56.0)$ & $52.0(43.2-60.0)$ \\
\hline Gender (M:F) & $92: 25$ & $22: 4$ & $70: 21$ \\
\hline$\%$ TBSA & $26.0(15.0-43.5)$ & $57.5(33.0-60.0)^{*}$ & $22.0(12.0-31.0)$ \\
\hline Inhalation burn (Y:N) & $46: 71$ & $18: 8^{*}$ & $28: 63$ \\
\hline Full thickness burn (Y:N) & $62: 54$ & $16: 10$ & $46: 44$ \\
\hline Mode Flame/Electric/Scalding/Chemical/other & $68 / 20 / 18 / 8 / 3$ & $21 / 0 / 2 / 3 / 0$ & $47 / 20 / 16 / 5 / 3$ \\
\hline SAP-3 score & $32.0(27.0-38.5)$ & $33.0(30.0-39.0)$ & $31.5(25.0-37.0)$ \\
\hline Day of ICU stay & $9.0(4.0-29.5)$ & $26.0(15.5-52.0)^{*}$ & $7.0(3.3-20.8)$ \\
\hline Death $(Y: N)$ & 25:93 & $11: 16^{*}$ & $14: 77$ \\
\hline $\mathrm{IG} \%$ & $2.6(0.8-5.2)$ & $6.4(3.1-11.6)^{*}$ & $1.4(0.6-4.4)$ \\
\hline IG count $\left(\times 10^{9} / \mathrm{L}\right)$ & $0.34(0.07-0.85)$ & $0.64(0.30-2.02)^{*}$ & $0.21(0.06-0.63)$ \\
\hline WBC $\left(\times 10^{9} / \mathrm{L}\right)$ & $11.8(7.8-15.8)$ & $10.4(4.5-15.2)$ & $12.0(8.4-16.0)$ \\
\hline Absolute neutrophil count $\left(\times 10^{9} / \mathrm{L}\right)$ & $9.0(5.4-12.0)$ & $8.1(3.8-11.7)$ & $9.4(5.7-12.2)$ \\
\hline Procalcitonin $(\mu \mathrm{g} / \mathrm{L})$ & $1.18(0.52-4.19)$ & $1.72(0.69-5.47)$ & $0.82(0.31-4.17)$ \\
\hline $\mathrm{CRP}(\mathrm{mg} / \mathrm{L})$ & $103.6(41.7-173.6)$ & $176.5(148.6-221.8)^{*}$ & $77.8(33.2-143.6)$ \\
\hline Lactate (mmol/L) & $3.3(2.2-5.1)$ & $4.9(3.5-6.4)^{*}$ & $2.8(2.2-4.7)$ \\
\hline Positive blood Culture (\%) & $39(33.3)$ & $26(100)^{*}$ & $13(14.3)$ \\
\hline Total organisms detected number (\%) & 65 & 47 & 18 \\
\hline Acinetobacter baumannii & $24(36.9)$ & $16(34.0)$ & $8(44.4)$ \\
\hline CRAB & $6(9.2)$ & $4(8.5)$ & $2(11.1)$ \\
\hline Pseudomonas aeruginosa & $12(18.5)$ & $9(19.1)$ & $3(16.7)$ \\
\hline CRPA & $4(6.2)$ & $3(6.4)$ & $1(5.6)$ \\
\hline Enterococcus spp. & $5(7.7)$ & $4(8.5)$ & $1(5.6)$ \\
\hline Klebsiella pneumoniae & $4(6.2)$ & $4(8.5)$ & 0 \\
\hline MRSA & $3(4.6)$ & $2(4.3)$ & $1(5.6)$ \\
\hline Proteus mirabilis & $2(3.1)$ & $2(4.3)$ & 0 \\
\hline Escherichia coli & $2(3.1)$ & $1(2.1)$ & $1(5.6)$ \\
\hline Others & $3(4.6)$ & $2(4.3)$ & $1(5.6)$ \\
\hline Antibiotics use & $93(79.5)$ & $26(100)$ & $67(73.6)$ \\
\hline
\end{tabular}

Continuous data are quoted as median values with interquartile range

*P $<0.05$, Data are compared using a Mann-Whitney or Chi-square test between "sepsis" and "non- sepsis"

IG immature granulocyte, TBSA total body surface area, ICU intensive care unit, SAPS-3 simplified acute physiology score-3, WBC white blood cell, CRP c-reactive protein, CRAB Carbapenem-resistant Acinetobacter baumannii, CRPA Carbapenem-resistant Pseudomonas aeruginosa, MRSA Methicillin-resistant Staphylococcus aureus

\section{CRP, lactate, and procalcitonin}

CRP and lactate levels [IQR] were higher in the sepsis group than in the non-sepsis group (CRP: $176.5 \mathrm{mg} / \mathrm{L}$ [148.6-221.8] vs. $77.8 \mathrm{mg} / \mathrm{L}$ [33.2-143.6] and lactate: $4.9 \mathrm{mmol} / \mathrm{L}$ [3.5-6.4] vs. $2.8 \mathrm{mmol} / \mathrm{L}$ [2.2-4.7], respectively, all $P<0.001)$. Procalcitonin levels were also higher in the sepsis group than in the non-sepsis group, but the difference was not statistically significant.

\section{Correlation between WBC count and IG\%}

The IG\% in the sepsis group was also higher overall than that in the non-sepsis group, both for patients with a WBC count $<4.0 \times 10^{9} / \mathrm{L}$ and those with a normal WBC count (Fig. 2). For patients with a WBC count $<4.0 \times 10^{9} / \mathrm{L}, 4.0-10.0 \times 10^{9} / \mathrm{L}$, and $>10.0 \times 10^{9} / \mathrm{L}$ in the sepsis group, the IG\% was $5.5 \%(1.0-12.0 \%), 8.0 \%(6.0-11.0 \%)$, and $5.5 \%$ (3.0-16.0), respectively $(P=0.823)$. For patients with a WBC count $<4.0 \times 10^{9} / \mathrm{L}, 4.0-10.0 \times 10^{9} / \mathrm{L}$, and $>10.0 \times 10^{9} / \mathrm{L}$ in the non-sepsis group, the IG\% was $3.0 \%(1.0-5.10 \%), 1.0 \%(0.0-2.0 \%)$, and $3.0 \%(2.0-$ $8.0 \%)$, respectively $(P=0.065)$.

\section{ROC curve analysis}

The ROC curve analysis was performed to evaluate the differences in the sensitivities and specificities of the IG\% and biomarkers in the sepsis and non-sepsis groups. The AUC was highest for CRP (0.82, 95\% CI: 0.74-0.89), followed by IG\% $(0.77,95 \%$ CI: $0.68-0.84)$, lactate $(0.73$, 95\% CI: 0.64-0.81), and procalcitonin (0.610, 95\% CI: 0.46-0.74). The AUC of combined IG\% and CRP showed 


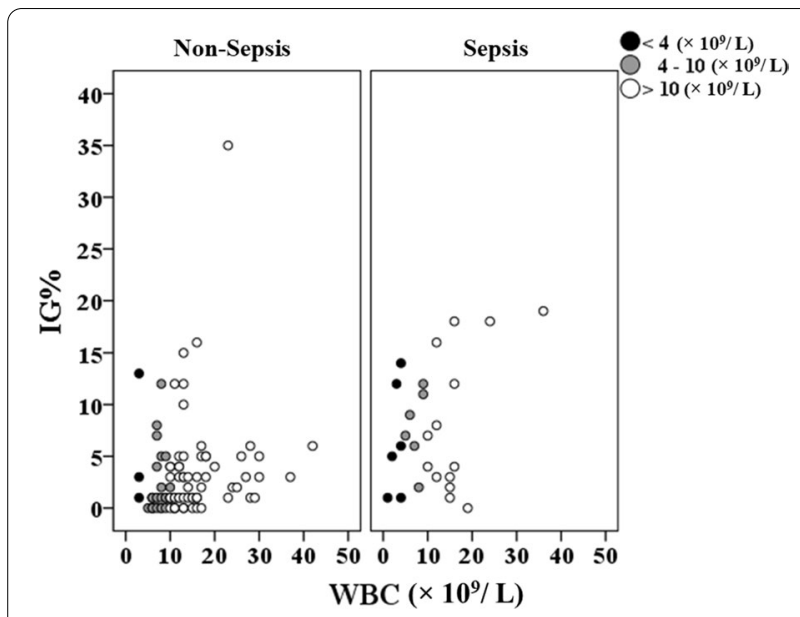

Fig. 2 Distribution of IG\% as related to the WBC count in the sepsis and non-sepsis groups. The patient groups were divided into 3 subgroups based on the WBC count $\left(<4 \times 10^{9} / \mathrm{L} ; 4-10 \times 10^{9} / \mathrm{L}\right.$; and $>10 \times 10^{9} / \mathrm{L}$

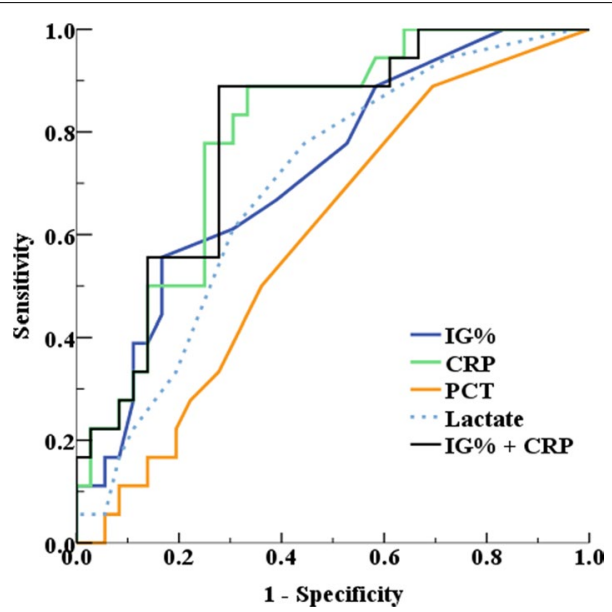

Fig. 3 Receiver operator characteristic (ROC) curve to evaluate the biomarkers for diagnosis of sepsis in burns. IG\% immature granulocyte percentage, CRP C-reactive protein, $P C T$ procalcitonin

higher AUC (0.85, 95\% CI: 0.78-0.92), compared to that of CRP or IG\% alone. The optimal cut-off value for the IG\% was $3 \%$, with a sensitivity of $76.9 \%$ (95\% CI:
56.4-91.0) and specificity of $68.1 \%$ (95\% CI: 57.5-77.5) (Fig. 3, Table 3).

\section{Logistic regression analysis}

We analyzed the predictive power of the IG\% and other biomarkers for the diagnosis of sepsis using logistic regression analysis. The odds ratio $[95 \% \mathrm{CI}]$ for IG\% (1.15 [1.05-1.25]), CRP (1.01 [1.01-1.02]), and lactate $(1.22[1.07-1.39])$ were found to be significant (Table 4). We further analyzed the significant variables using multivariate logistic regression with known risk factors (age, \%TBSA, and presence of inhalation burns), which revealed that the odds ratio [95\% CI] for IG\% (1.09 [1.00$1.08])$ and CRP (1.02 [1.03-1.08]) were significantly associated with sepsis.

\section{Machine learning application for evaluating the IG\% PDP for sepsis prediction}

Figure 4 shows the single-predictor PDP of each variable's predictive value for predicting sepsis, including the IG\%, CRP, \%TBSA, lactate, age, and procalcitonin. In this visual plot, the probability of developing sepsis was found to increase significantly as the values of IG\%, CRP, and \%TBSA increased. The PDP showed that an IG\% $<4 \%$ had a low probability of sepsis that increased thereafter. For CRP and \%TBSA, the probability plot increased sharply above $140 \mathrm{mg} / \mathrm{L}$ and $30 \%$, respectively. The probability of developing sepsis increased as lactate levels exceeded $2.5 \mathrm{mmol} / \mathrm{L}$, but the change was not as sharp. Sepsis probability decreased with age such that those aged 20-25 years had the highest predicted sepsis risk. For procalcitonin, the probability of sepsis did not change significantly.

\section{ICE plot for sepsis probability according to IG\%}

On the ICE plot for IG\%, the centered line (mean), starting at 0 for sepsis probability, increased rapidly after an IG\% of $4 \%$. The other lines (individual patients) showed similar patterns, with most patients showing an increase in predicted sepsis probability with increasing IG\% (Fig. 5).

Table 3 Receiver operating characteristic analysis of IG\%, PCT, CRP, and lactate for discriminate the sepsis

\begin{tabular}{llcll}
\hline Biomarker & \multicolumn{1}{l}{ Sepsis } & & \\
\cline { 2 - 5 } & AUC $\mathbf{( 9 5 \% ~ C l )}$ & Optimal cutoff & Sensitivity $\mathbf{( 9 5 \% ~ C l )}$ & Specificity (95\% Cl) \\
\hline IG\% & $0.77(0.68,0.84)$ & 3.0 & $76.9(56.4,91.0)$ & $68.1(57.5,77.5)$ \\
CRP & $0.82(0.74,0.89)$ & 15.3 & $76.0(54.9,90.6)$ & $79.3(69.3,87.3)$ \\
Lactate & $0.73(0.64,0.81)$ & 3.1 & $88.5(69.8,97.6)$ & $59.1(48.1,69.5)$ \\
Procalcitonin & $0.61(0.46,0.74)$ & 0.32 & $94.4(72.7,99.9)$ & $30.6(16.3,48.1)$ \\
\hline
\end{tabular}


Table 4 Logistic regression analysis for biomarkers predicting sepsis

\begin{tabular}{|c|c|c|c|c|}
\hline Variables & Univariate analysis, OR $(95 \% \mathrm{Cl})$ & $P$-value & $\begin{array}{l}\text { Multivariate analysis*, OR (95\% } \\
\text { Cl) }\end{array}$ & $P$-value \\
\hline Age (year) & $0.98(0.96-1.01)$ & 0.263 & & \\
\hline Inhalation burn & $2.02(1.19-3.41)$ & 0.009 & & \\
\hline TSBA\% & $1.05(1.02-1.07)$ & $<0.001$ & & \\
\hline $\mathrm{IG} \%$ & $1.15(1.05-1.25)$ & 0.002 & $1.09(1.00-1.18)$ & 0.043 \\
\hline Procalcitonin $(\mu \mathrm{g} / \mathrm{L})$ & $0.99(0.95-1.03)$ & 0.666 & & \\
\hline $\mathrm{CRP}(\mathrm{mg} / \mathrm{L})$ & $1.02(1.01-1.02)$ & $<0.01$ & $1.02(1.03-1.08)$ & $<0.001$ \\
\hline lactate $(\mathrm{mmol} / \mathrm{L})$ & $1.16(1.01-1.33)$ & 0.032 & $1.06(0.88-1.26)$ & 0.542 \\
\hline
\end{tabular}

*Multivariate analysis adjusted for age, TBSA, and inhalation injury

\section{Multi-predictor PDP of sepsis probability}

The multi-predictor PDP of sepsis probability and the interaction between IG\% and \%TBSA and between IG\% and CRP are described in Fig. 6A, B, respectively. The plot shows an increase in sepsis probability at an IG\% of $5 \%$ or $6 \%$. For an IG\% above $6 \%$, patients with $>60 \%$ TBSA or a CRP $>160 \mathrm{mg} / \mathrm{L}$ had a higher predicted sepsis risk that was near to or greater than 0.4 , which was higher than the predictability of any single parameter alone.

\section{Discussion}

In this study, IG\% showed moderate predictability for sepsis in patients with severe burns. There was no significant difference in WBC count or absolute neutrophil count between the sepsis and non-sepsis groups; however, while the non-sepsis group showed a slightly higher IG count, the IG\% was significantly higher in the sepsis group. CRP and lactate levels were higher in the sepsis group than in the non-sepsis group; however, the differences in the percentages were not as large as those for the IG\%. The usefulness of the IG\% was also demonstrated using the ROC curve and logistic regression analysis. The IG\% had the second-highest AUC after CRP, indicating that this parameter showed good discrimination between the two groups. Logistic regression analysis showed that the IG\% was significantly associated with the development of sepsis.

Our results are consistent with those of previous studies. Porizka et al. [13] reported that IG\% was significantly higher in cardiac surgical patients with sepsis than in those with non-infective SIRS, indicating that IG\% is a helpful marker with a moderate ability to discriminate between sepsis and non-sepsis. Hampson et al. [14] showed that the IG count was elevated in septic patients with burn injuries. In our study, moreover, the relationship between IG\%, other biomarkers, and known risk factors for sepsis probability were analyzed using machine learning.
Machine learning algorithms have demonstrated the ability to automate the interpretation and analysis of laboratory data in a variety of fields [15] and have also been adapted in medical research in recent years. We used PDP and ICE plots to visualize the relationship between the predictors and sepsis predictability. PDP are lowdimensional graphical renderings of the prediction function and are especially useful for visualizing relationships discovered by complex machine learning algorithms. This also allows for any number of predictors to be assessed, and a multivariate display can thus be obtained. The PDP is the average of the lines of the ICE plot. ICE plots display one line per individual patients that shows how the patient's prediction changes when IG\% changes $[16,17]$.

The results of the single-predictor PDP showed that the IG\% significantly increased the probability of sepsis, after the \%TBSA and CRP. The sepsis probability was low until an IG\% of 3\% was reached but increased rapidly above 0.3 when the IG\% exceeded $4 \%$. This was consistent with the cut-off result of the ROC curve analysis. Interpreting the ICE for IG\% was much more intuitive and heterogeneous relationships were easily found. Similar to the pattern of the centered line, most patients showed little change in sepsis predictability up to an IG\% of $4 \%$. Since CRP also showed good predictability in the single-predictor PDP and was a significant factor along with IG\% in the ROC and logistic regression analyses, we used a multi-predictor PDP to show the effect of the interaction between CRP and IG\% on sepsis predictability. On this plot, when the IG\% increased above 6\% and the CRP level was above $160 \mathrm{mg} / \mathrm{L}$, the probability of sepsis increased significantly, above 0.4. Considering that none of the biomarkers assessed individually exceeded 0.4 for predictability, the application of the IG\% with CRP could significantly improve predictability.

Previous studies have reported on different aspects of the IG\% in relation to sepsis. Karon et al. [18] reported that the IG\% and IG count were of limited utility in predicting sepsis in a study of patients admitted to the 

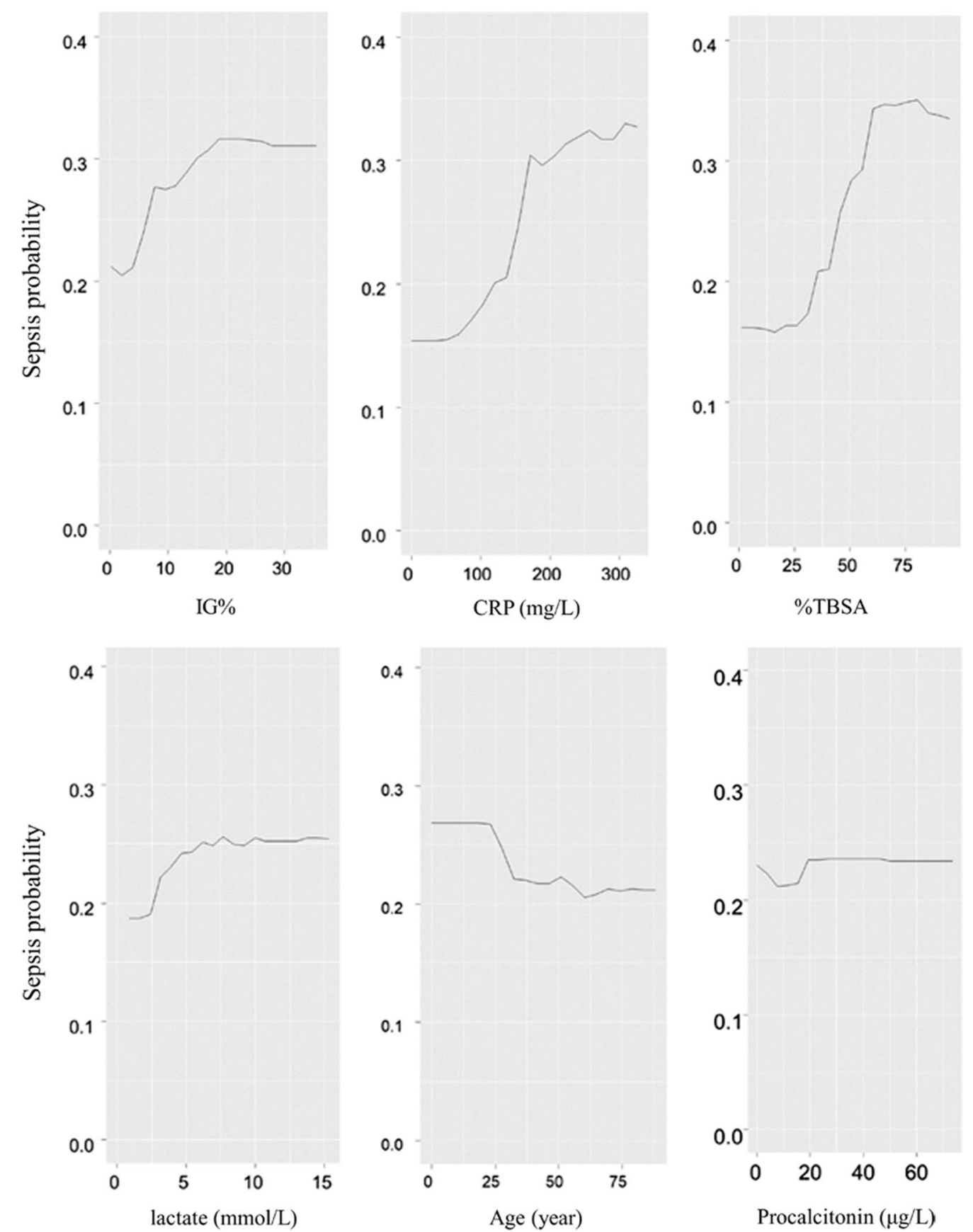

Fig. 4 Partial dependent plots (PDP) for sepsis prediction. For IG\%, the PDP showed that the probability was low until 4\%, and increased rapidly after. In CRP and TBSA, probability plot increased sharply above $140 \mathrm{mg} / \mathrm{L}$ and 30\%, respectively. Lactate's increased when above $2.5 \mathrm{mmol} / \mathrm{L}$ but showed less change. Sepsis probability decreased with age, age between 20 and 25 years-old had higher predicted sepsis risk. For procalcitonin, the sepsis probability does not change significantly. IG\% immature granulocyte percentage, CRP C-reactive protein, \%TBSA total body surface area percentage

emergency department. Additionally, unlike the findings of our study, Buoro et al. [19] reported that an IG $<10.0 \%$ was a valid cut-off for the diagnosis of sepsis in ICU patients. Ayres et al. [20] reported that an IG\% $<2.0 \%$ was adequate for excluding sepsis as a diagnosis. However, these previous studies included patients who had various underlying etiologies, while only patients with severe burns who were admitted to the BICU at a burn 


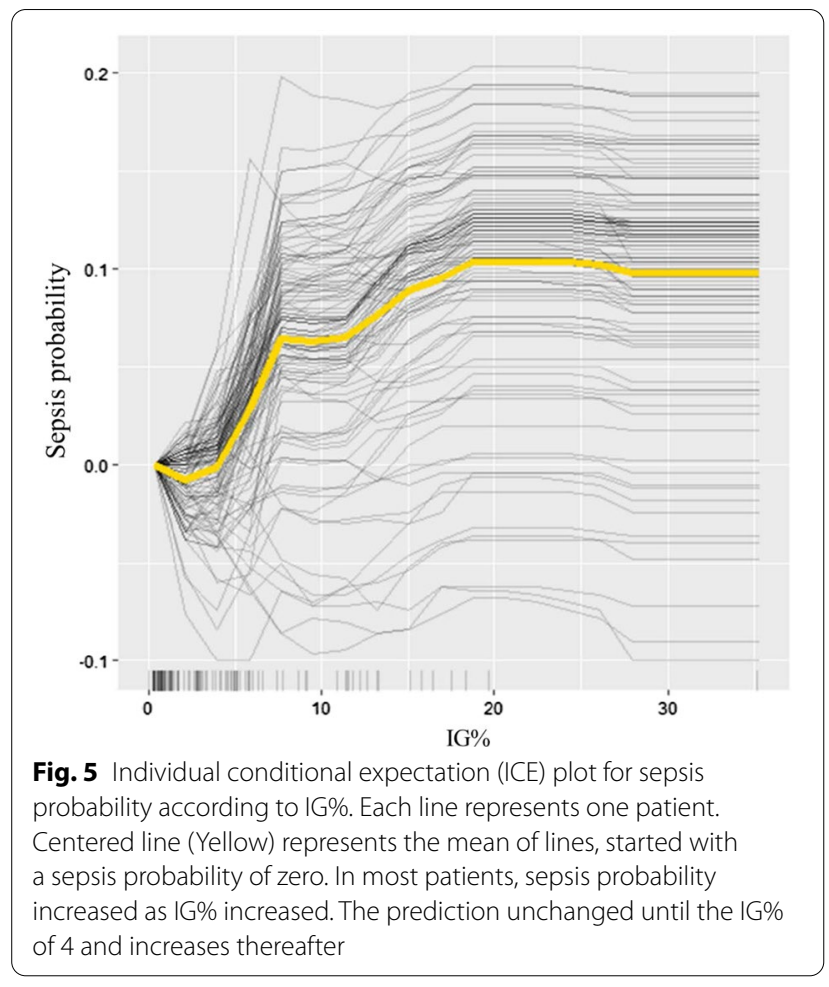

specialized center were included in our study to identify the discriminatory power of IG\% for patients with sepsis.

A few studies have been conducted on sepsis biomarkers in burn patients. Wineberg et al. [21] and Kundes et al. [22] studied procalcitonin in adults and children, respectively. In these studies, procalcitonin was shown to be a good marker for diagnosing sepsis in burn patients, but it was not effective in our study. The association between lactate and sepsis has also been assessed in several studies [23-25]. However, none of these studies have assessed sepsis in only burn patients. In our study, lactate appeared to have moderate discriminatory power according to the AUC, but the PDP analysis showed unclear predictability.

The advantage of using IG\% is that it can be obtained from a routine complete blood count, and therefore does not require further laboratory protocols, while other biomarkers require additional time, procedures, and costs. Another advantage is that the IG\% tends to be high in sepsis regardless of the WBC count. Patients with sepsis may not have an increase in neutrophils, and may even be neutropenic. In these situations, an increase in the IG\% can be useful for identifying an acute infection, even when not suspected $[5,26]$. Our results showed that the IG\% was higher in the sepsis group, even when the WBC count was low or normal. It may therefore serve as an independent marker that can be used in cases of sepsis showing leukocytopenia.

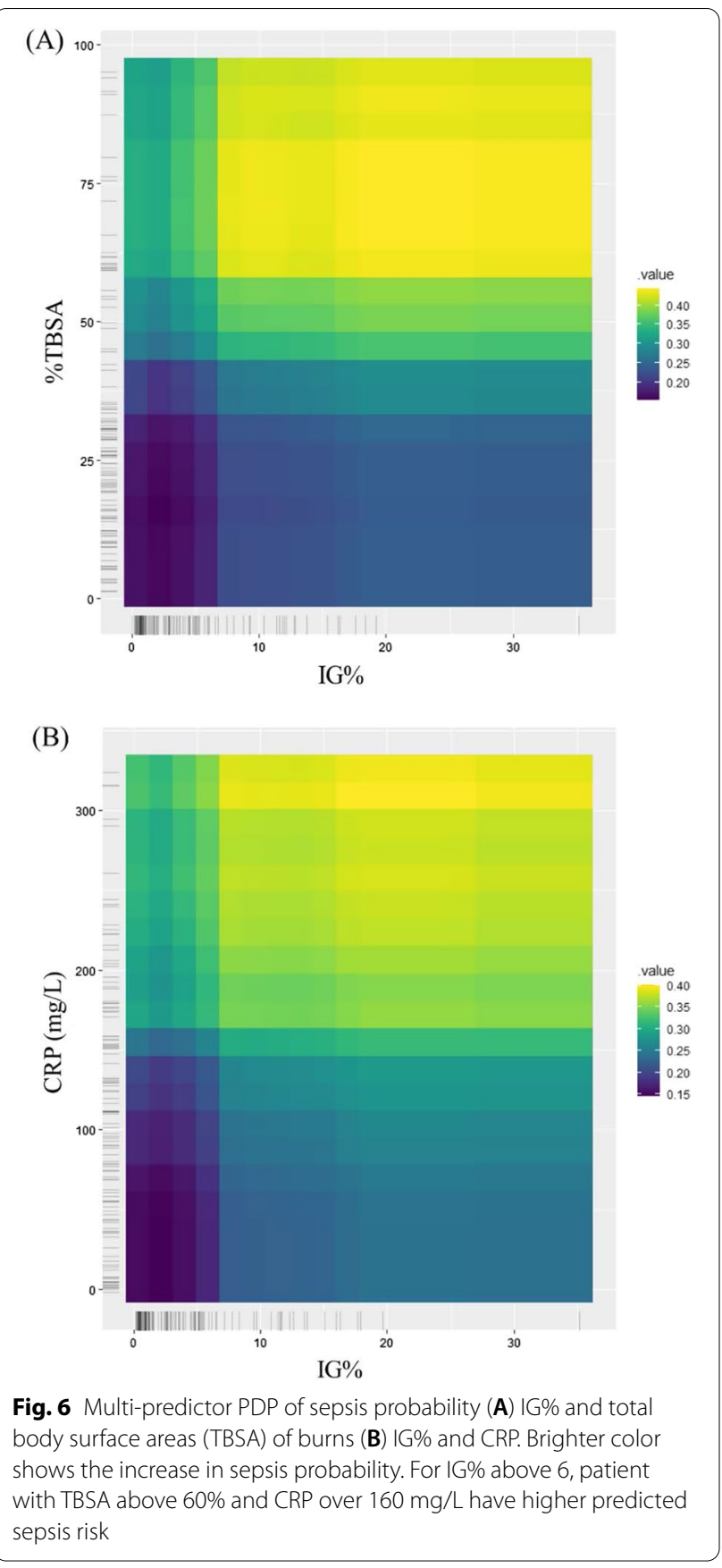

Our study was unique in the following aspects: (1) the relationship between parameters and predictability was identified by applying a machine learning approach, and these visualized results increased reliability and allowed for us to review findings from different aspects; (2) the study was performed at a single center and only severe burn patients in a BICU were included, which helped minimize possible institutional or instrument-dependent biases; (3) our study provided an IG\% cut-off value using 
a ROC curve analysis as well as machine learning technique to demonstrate its clinical applicability as an independent indicator of sepsis; and (4) a cut-off value for the CRP level and IG\% were determined, increasing the coutility of IG\% and as a sepsis biomarker.

Our study also has several limitations. The number of patients involved was small. There was also a difference in the type of burns and \%TBSA, which could have affected the distribution of the granulocyte indices. In addition, it would be difficult to apply our results to all age groups, as newborns have a higher IG\% than adults [6]. Finally, the discriminatory power and the predictability of the IG\% were not high, so it is insufficient to be used as a standalone biomarker for sepsis. CRP is a well-known, widely studied biomarker in sepsis. It showed better discriminatory power and the predictability than IG\% in our study. As shown by multivariate ROC and PDP results, discriminatory power and the predictability were higher when CRP and IG\% are combined than each alone. Therefore, IG\% results can be useful when assessed as an auxiliary to CRP.

\section{Conclusions}

In conclusion, IG\% is moderately useful for predicting sepsis in patients with severe burns. We used a machine learning approach to predicting, observed an increase in the probability of developing sepsis when IG and CRP were higher than $6 \%$ and $160 \mathrm{mg} / \mathrm{L}$, respectively. Our results emphasize that the IG\% is useful as an auxiliary marker, can be applied quickly and easily without requiring additional procedures or costs to assist in the prevention of sepsis progression in patients with severe burns who have life-threatening conditions.

\begin{abstract}
Abbreviations
SIRS: Systemic inflammatory response syndrome; IG: Immature granulocytes; BICU: Burn intensive care unit; TBSA: Total body surface area; CRP: C-reactive protein; PDP: Partial dependence plot; ICE: Individual conditional expectation; SAPS-3: Simplified Acute Physiology Score-3; CRAB: Carbapenem-resistant Acinetobacter baumannii; CRPA: Carbapenem-resistant Pseudomonas aeruginosa; MRSA: Methicillin-resistant Staphylococcus aureus.
\end{abstract}

\section{Supplementary Information}

The online version contains supplementary material available at https://doi. org/10.1186/s12879-021-06971-2.

Additional file 1. Supplementary Figure 1. Schematic diagram of the machine learning process for prediction of sepsis task.

\section{Acknowledgements}

Not applicable.

\section{Author contributions}

$\mathrm{KJ}$ performed the statistical analyses, analyzed the results, and was a major contributor in writing the manuscript. SJ, MJP, and WKS participated in the study design and directed the study. NL participated in the study design and manuscript editing and performed and verified the statistical analyses. The authors have read and approved the final manuscript.

\section{Funding}

None.

\section{Availability of data and materials}

All data generated or analyzed during this study are included in this published article.

\section{Declarations}

\section{Ethics approval and consent to participate}

The study was approved by the institutional review board of the Hallym University Hangang Sacred Hospital (IRB no. HG2018-069), which waived the requirement for written informed consent owing to the observational and anonymized nature of the study. The study was performed in accordance with the guidelines of the Declaration of Helsinki.

\section{Consent for publication}

Not applicable.

\section{Competing interests}

The authors declare that they have no competing interests.

\section{Author details}

${ }^{1}$ Department of Laboratory Medicine, Hallym University Hangang Sacred Heart Hospital, 12 Beodeunaru-ro 7-gil, Yeongdeungpo-gu, Seoul 150719, South Korea. ${ }^{2}$ Department of Laboratory Medicine, Hallym University Kangnam Sacred Heart Hospital, Hallym University College of Medicine, 22, Singil-ro 1, Yeongdeungpo-gu, Seoul 07440, South Korea.

Received: 11 August 2021 Accepted: 13 December 2021

Published online: 16 December 2021

\section{References}

1. Greenhalgh DG. Sepsis in the burn patient: a different problem than sepsis in the general population. Burns Trauma. 2017:5:23.

2. Gyawali B, Ramakrishna K, Dhamoon AS. Sepsis: the evolution in definition, pathophysiology, and management. SAGE Open Med. 2019. https://doi.org/10.1177/2050312119835043.

3. Vincent JL. The clinical challenge of sepsis identification and monitoring. PLoS Med. 2016. https://doi.org/10.1371/journal.pmed.1002022.

4. Ansari-Lari MA, Kickler TS, Borowitz MJ. Immature granulocyte measurement using the Sysmex XE-2100: relationship to infection and sepsis. Am J Clin Pathol. 2003;120:795-9.

5. Briggs C, Kunka S, Fujimoto H, Hamaguchi Y, Davis BH, Machin SJ. Evaluation of immature granulocyte counts by the XE-IG master: upgraded software for the XE-2100 automated hematology analyzer. Lab Hematol. 2003;9:117-24.

6. Wiland EL, Sandhaus LM, Georgievskaya Z, Hoyen CM, O'Riordan MA, Nock ML. Adult and child automated immature granulocyte norms are inappropriate for evaluating early-onset sepsis in newborns. Acta Paediatr. 2014;103:494-7.

7. Ha SO, Park SH, Park SH, Park JS, Huh JW, Lim CM, et al. Fraction of immature granulocytes reflects severity but not mortality in sepsis. Scand J Clin Lab Investig. 2015;75:36-43.

8. Park SH, Park CJ, Lee BR, Nam KS, Kim MJ, Han MY, et al. Sepsis affects most routine and cell population data (CPD) obtained using the Sysmex XN-2000 blood cell analyzer: neutrophil-related CPD NE-SFL and NE-WY provide useful information for detecting sepsis. Int J Lab Hematol. 2015;37:190-8. 
9. Honda T, Uehara T, Matsumoto G, Arai S, Sugano M. Neutrophil left shift and white blood cell count as markers of bacterial infection. Clin Chim Acta. 2016:457:46-53.

10. Ishimine N, Honda T, Yoshizawa A, Kawasaki K, Sugano M, Kobayashi $Y$, et al. Combination of white blood cell count and left shift level real-timely reflects a course of bacterial infection. J Clin Lab Anal. 2013;27:407-11.

11. Greenhalgh DG, Saffle JR, Holmes JH, Gamelli RL, Palmieri TL, Horton JW et al. American Burn Association consensus conference to define sepsis and infection in burns. J Burn Care Res. 2007;28:776-90.

12. Maenhout TM, Marcelis L. Immature granulocyte count in peripheral blood by the Sysmex haematology XN series compared to microscopic differentiation. J Clin Pathol. 2014;67:648-50.

13. Porizka M, Volny L, Kopecky P, Kunstyr J, Waldauf P, Balik M. Immature granulocytes as a sepsis predictor in patients undergoing cardiac surgery. Interact Cardiovasc Thorac Surg. 2019;28:845-51.

14. Hampson P, Dinsdale RJ, Wearn CM, Bamford AL, Bishop JRB, Hazeldine J, et al. Neutrophil dysfunction, immature granulocytes, and cell-free DNA are early biomarkers of sepsis in burn-injured patients: a prospective observational cohort study. Ann Surg. 2017:265:1241-9.

15. Friedman JH. Greedy function approximation: a gradient boosting machine. Ann Stat. 2001;29:1189-232.

16. Greenwell BM. pdp: an R package for constructing partial dependence plots. R J. 2017:9:421-36.

17. Elshawi R, Al-Mallah MH, Sakr S. On the interpretability of machine learning-based model for predicting hypertension. BMC Med Inform Decis Mak. 2019:19:146.

18. Karon BS, Tolan NV, Wockenfus AM, Block DR, Baumann NA, Bryant SC, et al. Evaluation of lactate, white blood cell count, neutrophil count, procalcitonin and immature granulocyte count as biomarkers for sepsis in emergency department patients. Clin Biochem. 2017:50:956-8.

19. Buoro S, Mecc T, Vavasso M, Azza G, Espos SA, Dominoni P, et al. Immature granulocyte count on the new Sysmex XN-9000: performance and diagnosis of sepsis in the intensive care unit. Signa Vitae. 2015;10:54-65.

20. Ayres LS, Sgnaolin V, Munhoz TP. Immature granulocytes index as early marker of sepsis. Int J Lab Hematol. 2019;41:392-6.

21. Wineberg D, Moore R, Kruger D. Procalcitonin and bacterial sepsis in burn patients in South Africa. J Surg Res. 2020;246:490-8.

22. Kundes MF, Kement M. Value of procalcitonin levels as a predictive biomarker for sepsis in pediatric patients with burn injuries. Niger J Clin Pract. 2019;22:881-4.

23. Ryoo SM, Lee J, Lee YS, Lee JH, Lim KS, Huh JW, et al. Lactate level versus lactate clearance for predicting mortality in patients with septic shock defined by Sepsis-3. Crit Care Med. 2018;46:489-95.

24. Liu Z, Meng Z, Li Y, Zhao J, Wu S, Gou S, et al. Prognostic accuracy of the serum lactate level, the SOFA score and the GSOFA score for mortality among adults with sepsis. Scand J Trauma Resusc Emerg Med. 2019;27:51.

25. Jaiswal P, Dewan P, Gomber S, Banerjee BD, Kotru M, Malhotra RK, et al. Early lactate measurements for predicting in-hospital mortality in paediatric sepsis. J Paediatr Child Health. 2020;56:1570-6.

26. Subramanian A, Agrawal D, Pandey RM, Nimiya M, Albert V. The leukocyte count, immature granulocyte count and immediate outcome in head injury patients. Brain Injury—-Pathogenesis, Monitoring, Recovery and Management. 2011; https://doi.org/10.5772/27020.

\section{Publisher's Note}

Springer Nature remains neutral with regard to jurisdictional claims in published maps and institutional affiliations. 